\title{
CMAJ
}

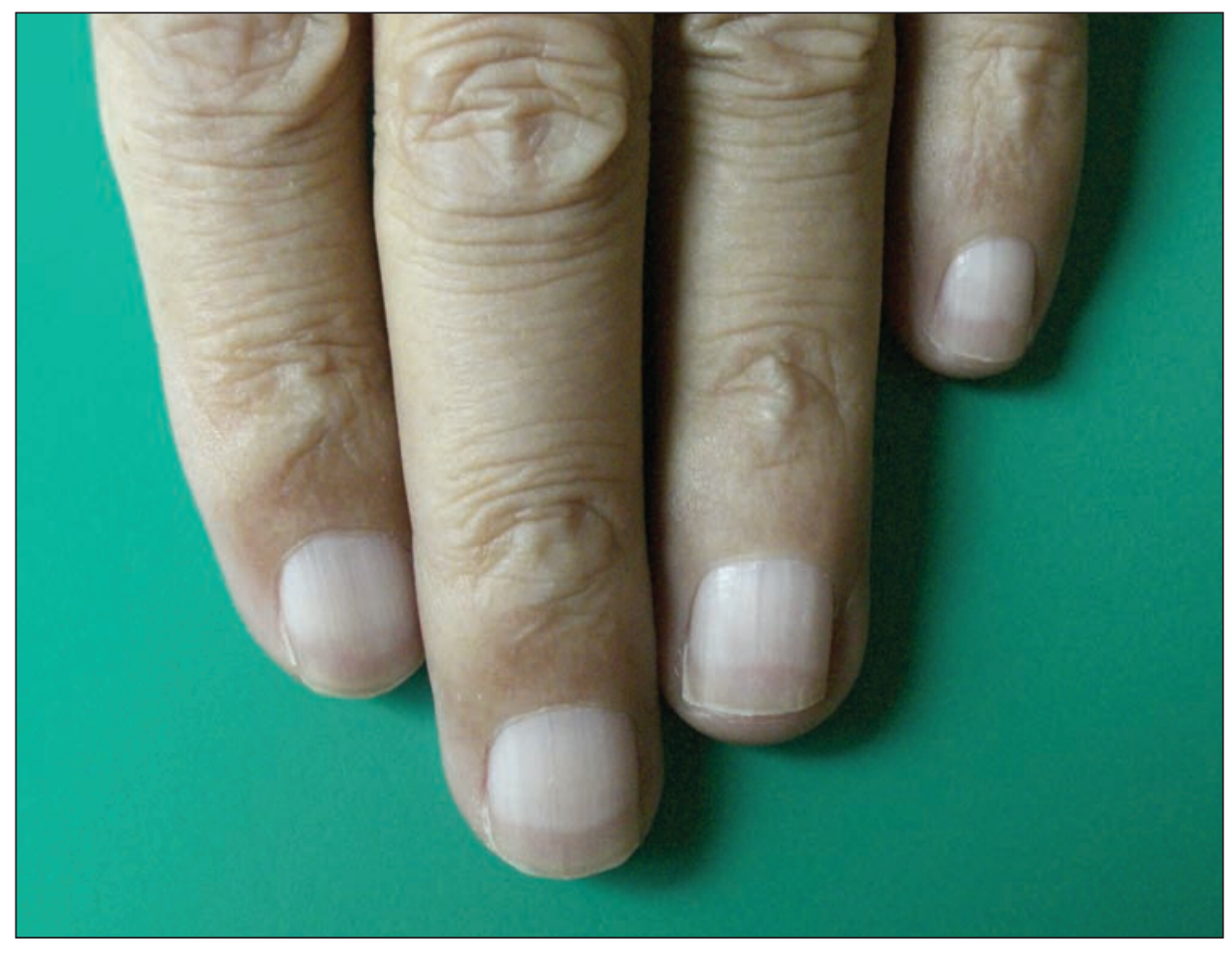

Figure 1: Photograph of the fingernails of a 61-year-old man with chronic renal failure, showing distal pink transverse bands with sharp borders and diffuse whitening of proximal nailbeds.

\section{Half-and-half nail}

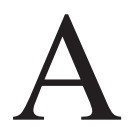

61-year-old man with diabetes mellitus and chronic kidney disease presented with a 2-week history of nausea, vomiting and general malaise. Laboratory tests showed he had a hemoglobin level of 72 (normal 138-172) g/L, a serum urea level of 38.2 (normal 2.9-8.9) $\mathrm{mmol} / \mathrm{L}$, a serum creatinine level of 1229 (normal 62-133) $\mu \mathrm{mol} / \mathrm{L}$ and a blood glucose level of 8.69 (normal 3.58-6.00) mmol/L. On examination, his fingernails had a distinctive pinkish red transverse band distally and diffuse, dull whitening of the proximal nailbeds (Figure 1). The margin between the 2 zones was sharp even after venous return was constricted, a characteristic of half-and-half nail.

Half-and-half nail is an occasional but specific clinical finding in chronic renal failure. It affects fingernails and, less often, toenails. The distal $20 \%-60 \%$ of the nail is red, pink or brown. The rest of the nail has a dull, whitish, ground-glass appearance. ${ }^{1}$ No correlation exists between the severity of azotemia and the depth of the distal colour band. The band usually remains unchanged even after dialysis but may disappear after successful renal transplantation. The pathogenesis of half-and-half nail is unclear. Estimates of prevalence among patients with chronic renal failure range from $15 \%$ to $50 \%{ }^{1,2}$ Routine inspection of the nails is an essential part of the physical examination to check for possible azotemia.
Clinical images are chosen because they are particularly intriguing, classic or dramatic.

Submissions of clear, appropriately labelled high-resolution images must be accompanied by a figure caption and the patient's written consent for publication. A brief explanation (300 words maximum) of the educational significance of the images with minimal references is required.
Wen-Te Huang MD

Department of Medicine Zuoying Armed Forces

General Hospital

Chia-Chao Wu MD PhD

Division of Nephrology

Department of Medicine

Tri-Service General Hospital

National Defense Medical Center Taipei, Taiwan

\section{REFERENCES}

1. Lindsay PG. The half-and-half nail Arch Intern Med 1967;119:583-7.

2. Saray Y, Seckin D, Gulec AT, et al. Nail disorders in hemodialysis patients and renal transplant recipients: a casecontrol study. J Am Acad Dermatol 2004;50:197-202 